\title{
A study of patient experience and perception regarding postoperative pain management in Chinese hospitals
}

This article was published in the following Dove Press journal:

Patient Preference and Adherence

7 November 2013

Number of times this article has been viewed

\author{
Liu Weiran ${ }^{1, *}$ \\ Zhang Lei ${ }^{2, *}$ \\ Stephanie Mu-Lian Woo ${ }^{3, *}$ \\ Tang Anliu ${ }^{4, *}$ \\ Xie Shumin ${ }^{4, *}$ \\ Zhang Jing,* \\ Zhang Kai ${ }^{6, *}$ \\ Zhang Zhen ${ }^{7}$ \\ 'Department of Anesthesia, \\ ${ }^{2}$ Department of Thoracic Surgery, \\ Key Laboratory of Cancer Prevention \\ and Therapy, Tianjin Lung Cancer \\ Center, Tianjin Medical University \\ Cancer Institute and Hospital, \\ Tianjin, People's Republic of China; \\ ${ }^{3}$ Harvard University, Cambridge, MA, \\ USA $;{ }^{4}$ Xiangya Medical School of \\ Central South University, Changsha, \\ People's Republic of China; ${ }^{5}$ Tianjin \\ Medical University, Tianjin, People's \\ Republic of China; ${ }^{6}$ Department of \\ Cerebral Surgery, Tumor Hospital \\ Xiangya School of Medicine, Central \\ South University, Changsha, People's \\ Republic of China; ${ }^{7}$ Department of \\ Oncology, Third Xiangya Hospital, \\ Central South University, Changsha, \\ People's Republic of China \\ *These authors contributed equally \\ to this work
}

Correspondence: Zhang Zhen

Department of Oncology,

Third Xiangya Hospital of Central

South University, Changsha 410000,

People's Republic of China

Tel +867318638888

$\mathrm{Fax}+867318638888$

Email zhangzhen0819@gmail.com
Background: This study aims to analyze the current status of postoperative pain management in the People's Republic of China's provincial-level hospitals, and the existing knowledge and opinions held by patients regarding these methods.

Methods: The 128 participants in this study were urology and hepatobiliary patients from three provincial-level hospitals in Hunan. The questionnaire assessing postoperative pain was designed using the typical pain assessment scales and pain management guidelines as references.

Results: $82.8 \%$ of study participants claimed that their postoperative pain was relieved within 3 days of their operations. However, while $91.4 \%$ of surveyed patients experienced moderate to severe pain, $51.6 \%$ received no treatment for their postoperative pain, and $14.9 \%$ complained that medical personnel failed to manage their pain. $20.2 \%$ were unsatisfied with their pain management, indicating that treatment did not meet their expectations. Furthermore, participants demonstrated a great misunderstanding of pain and analgesics, as $72.6 \%$ of patients were unfamiliar with morphine, $51.6 \%$ of patients believed only certain types of pain required management, and $18.5 \%$ refused to use morphine.

Conclusion: In most Chinese provincial-level hospitals, current postoperative pain management methods are able to alleviate the pain experienced by the majority of patients, though pain assessment and therapy procedures are still not standardized. Furthermore, most patients lack a proper understanding of postoperative pain and analgesics. Therefore, pain management education for doctors and patients and their relatives should be implemented in order to improve the quality of postoperative pain management.

Keywords: postoperative pain, pain assessment, pain management, pain recognition, pain education

\section{Introduction}

According to the International Association for the Study of Pain, pain is an unpleasant sensory and emotional experience associated with actual or potential tissue damage or is described in terms of such damage. ${ }^{1}$ In clinical work, pain is receiving increasing attention and is regarded as the fifth vital sign, following temperature, pulse, respiration, and blood pressure. Acute postoperative pain has clinical, economic, and biological consequences, such as changes in immune system function, decreased healing, diminished ability to function, and needless suffering. ${ }^{2}$ Although postoperative pain management has improved in recent years, research shows that current methods are unsatisfactory. Maier et al investigated the quality of pain management for 2,252 postoperative patients in 25 German hospitals; $29.5 \%$ of surgical patients reported moderate to severe pain, and $55 \%$ of surgical patients were dissatisfied with 
their pain management. ${ }^{3}$ Meanwhile, a survey carried out in the United States revealed that approximately $80 \%$ of patients experienced pain after surgery, and of these patients, $86 \%$ had moderate, severe, or extreme pain. ${ }^{4}$

Currently in the People's Republic of China, the main pain management methods are patient-controlled analgesia (PCA) and temporary analgesics; however, little research has been done on methods of pain assessment and management in the People's Republic of China. In certain Chinese provincial-level hospitals, the McGill Pain Questionnaire, Numerical Rating Scale (NRS), etc have been adopted to assess the postoperative pain of patients. ${ }^{5,6}$ Despite the medical field's rising interest in postoperative pain, research on the current status of postoperative pain, public knowledge and opinions toward pain control, and studies with large samples remain absent in the People's Republic of China. In order to address these issues, this study investigates postoperative pain management in the People's Republic of China by surveying surgery patients on their experiences.

\section{Material and methods Postoperative patients}

Patients suffering from postoperative pain following urology or hepatobiliary surgery in the First, Second, and Third Xiangya Hospitals affiliated with Xiangya Medical School of Central South University were randomly selected for the study. The inclusion criteria were patient and family agreement to participate in the survey, minimum age of 9 years, functional physical condition, and mental ability to understand and answer survey questions.

\section{Methods}

A questionnaire assessing postoperative pain in the People's Republic of China was designed using the typical pain assessment scales, pain intensity rating scales, and pain management guidelines including McGill Pain Questionnaire, NRS, and Pain Management Guideline of the Health Care Association of New Jersey. ${ }^{5-7}$ The survey was conducted by nonanesthesia specialists and given to postoperative urology and hepatobiliary patients in three Hunan provincial-level hospitals. The questionnaire consisted of three parts: the first section assessed the patient's general and surgical conditions; the second section measured postoperative pain indicators including pain location, time, frequency, intensity, impact on quality of life (QOL), and treatment; the third section evaluated patient knowledge and opinion regarding pain management. Pain intensity was evaluated according to the NRS-11, and measured along a $0-10$ scale, where 0 is no pain,
$1-3$ is mild pain, 4-6 is moderate pain, and 7-10 is severe pain. The patients' level of pain intensity was recorded once every 8 hours for 4 days after surgery, beginning at the point of postanesthetic recovery; if a patient was discharged during the study, their survey was terminated.

\section{Statistical analyses}

All data was analyzed using SPSS version 13.0 software (SPSS Inc., Chicago, IL, USA). Age was expressed as mean \pm standard deviation, and the rate of postoperative pain related indicators was expressed as percentage. The Chi-square test was used to analyze two sample rates. A $P$-value of $<0.05$ was considered statistically significant.

\section{Ethics}

Prior to starting, this study was approved by the ethics committee at Xiangya Medical School of Central South University. Before taking the survey, all participants and guardians of child participants were informed of the study's objectives and signed an informed consent to confirm their willingness to take part in this study and acknowledged their rights to voluntary participation and privacy.

\section{Results}

\section{Patient general and surgical conditions}

One hundred and thirty six questionnaires were delivered and 128 were completed and retrieved 4 days later, with a valid answer rate of $94.1 \%$. Of eight invalid questionnaires, five participants quit unexpectedly and three participants failed to complete the questionnaire correctly. Among 128 participants, 75 were male, and 53 were female. The patients varied in age from 9-86 years, with a mean age of $45.73 \pm 15.57$ years. The operation categories are shown in Table 1.

\section{General conditions of postoperative pain}

The abdomen was identified as the most common incision site in operations ( $n=66,51.6 \%)$, and more than half of survey participants $(n=83,64.8 \%)$ experienced postoperative

Table I Operation categories $(n=128)$

\begin{tabular}{|c|c|c|c|}
\hline \multirow[t]{2}{*}{ Anesthesia } & \multicolumn{2}{|l|}{ Operation } & \multirow[t]{2}{*}{ Total } \\
\hline & $\begin{array}{l}\text { Open } \\
\text { operation }\end{array}$ & $\begin{array}{l}\text { Minimally } \\
\text { invasive operation }\end{array}$ & \\
\hline General anesthesia & 50 & 42 & 92 \\
\hline Epidural anesthesia & 12 & 6 & 18 \\
\hline $\begin{array}{l}\text { Combined spinal- } \\
\text { epidural anesthesia }\end{array}$ & 7 & 11 & 18 \\
\hline Total & 69 & 59 & 128 \\
\hline
\end{tabular}


abdominal pain. Furthermore, distending pain $(n=40,31.3 \%)$ and dull pain $(n=35,27.3 \%)$ accounted for the major kinds of pain. Some patients had pain at the site of the spinal anesthesia, presenting as distending pain, dull pain, or soreness. Most patients experienced no difference between pain in the morning and evening $(n=117,91.4 \%)$, and found that their pain mainly emerged as persistent $(n=73,57 \%)$ or irregular intermittent $(\mathrm{n}=47,36.7 \%)$.

\section{Negative effects of postoperative pain on patients' QOL}

This study found that postoperative pain resulted in varying consequences on patient QOL. Some patients with mild, moderate, or severe pain experienced debilitation, nausea, vomiting, dizziness, and headache without significant difference $(P>0.05)$. However, patients with moderate or severe pain were more likely to have sleep disorders, such as difficulty falling asleep, disturbed sleep as well as changes in mental activity including irritability, anxiety, anguish, depression, and fear $(P<0.05)$. Effects of postoperative pain on patient appetite, physical and social activity could not be assessed due to certain postoperative requirements, such as fasting and immobilization (Table 2).

\section{Postoperative pain management and its effects}

PCA and temporary analgesics were common pain management methods indicated by study participants. PCA is a method for the patient to self-administer analgesics intravenously by using a computerized pump, which sends specific doses into an intravenous line. If patients complain of pain immediately after surgery, during recovery, they are connected to a PCA pump with analgesics, such as fentanyl, sufentanil, and tramadol. The temporary analgesics recorded in this study

Table 2 Negative effects of postoperative pain on patients' quality of life

\begin{tabular}{llllll}
\hline $\begin{array}{l}\text { Harmful } \\
\text { effects }\end{array}$ & $\begin{array}{l}\text { Mild pain } \\
\mathbf{n}(\%)\end{array}$ & $\begin{array}{l}\text { Moderate or } \\
\text { severe pain } \\
\text { n (\%) }\end{array}$ & $\chi^{2}$ & df & P-value \\
\hline Debilitation & $3(25.0 \%)$ & $54(46.6 \%)$ & 2.045 & I & 0.153 \\
Nausea & $5(41.7 \%)$ & $35(30.2 \%)$ & 0.669 & I & 0.413 \\
Vomiting & $4(33.3 \%)$ & $29(25.0 \%)$ & 0.395 & I & 0.530 \\
Dizziness & $4(33.3 \%)$ & $25(21.6 \%)$ & $0.86 \mathrm{I}$ & $\mathrm{I}$ & 0.353 \\
Headache & $2(16.7 \%)$ & $20(17.2 \%)$ & 0.003 & $\mathrm{I}$ & 0.960 \\
Influence on & $4(33.3 \%)$ & $83(71.6 \%)$ & 7.296 & $\mathrm{I}$ & $0.007^{*}$ \\
sleep & & & & & \\
Mental change & $3(25.0 \%)$ & $86(74.1 \%)$ & 12.394 & $\mathrm{I}$ & $<0.00$ I $^{*}$ \\
\hline
\end{tabular}

Note: $* \mathrm{P}<0.05$.

Abbreviations: $d f$, degrees of freedom; $\chi^{2}$, Chi-square test. include nonopioids (tramadol), weak opioids (bucinperazine), and strong opioids (meperidine and fentanyl). Among the surveyed patients, 66 (51.6\%) remained untreated, 29 (22.7\%) used the PCA, and six (5.0\%) adopted a combination of PCA and analgesics to treat their postoperative pain (Table 3 ). Patients only received analgesic treatment once they could no longer bear their pain, and most patients received an irregular analgesic therapy. In one exception, a patient with intrahepatic cholangiocarcinomas used analgesic therapy regularly after undergoing an exploratory laparotomy and broad ligament biopsy. One hundred and six patients $(82.8 \%)$ claimed that the postoperative pain had disappeared or been relieved by the third day after the operation.

\section{Patient knowledge and opinion regarding pain management}

\section{Pain levels among patients prior to hospitalization}

One hundred and twenty four patient participants completed this section of the survey. Seventy four (59.7\%) respondents had suffered moderate pain ( $>3$ scores) before being hospitalized. Among these 74 patients, 18 sought medical help for mild pain, only 23 pursued treatment for severe pain, and four refrained from medical consultation altogether. Among the other 50 patients who did not suffer moderate or severe pain before hospitalization, 34 attempted to see a doctor for mild pain, and 16 expressed that they would seek treatment if suffering moderate pain. Moreover, a significant correlation was discovered between pain experiences and patient opinions regarding pain management $(P<0.001)$ Patients who had experienced severe pain prior to hospitalization felt stronger pain intensities when seeking pain management than those who had experienced only mild or moderate pain. This indicates that patients who had experienced severe pain in the past only sought treatment when they could no longer bear their pain. Before hospitalization, patients usually attempted to self-treat their pain, using analgesics $(n=60$, $48.4 \%)$, cheirapsis $(n=33,26.6 \%)$, and traditional Chinese medicine $(n=20,16.2 \%)$ (Table 4$)$.

Table 3 Postoperative pain management $(n=\mid 28)$

\begin{tabular}{lll}
\hline & $\mathbf{n}$ & $\%$ \\
\hline Without any treatments & 66 & 51.6 \\
PCA (fentanyl, sufentanil, tramadol) & 29 & 22.7 \\
Nonopioids & 23 & 18.0 \\
Weak opioids & 16 & 12.5 \\
Strong opioids & 4 & 3.1 \\
\hline Notes: 5\% of participants adopted PCA and temporary analgesics at the same time; \\
2.9\% used two kinds of opioids.
\end{tabular}

Patient Preference and Adherence 2013:7 
Table 4 Pain experience of patients before hospitalization $(n=124)$

\begin{tabular}{|c|c|c|c|c|c|c|}
\hline \multirow{2}{*}{$\begin{array}{l}\text { Suffering moderate } \\
\text { or severe pain } \\
\text { before in hospital }\end{array}$} & \multicolumn{2}{|c|}{$\begin{array}{l}\text { Pain intensity when consulting } \\
\text { for therapies } \mathrm{n}(\%)\end{array}$} & \multirow[t]{2}{*}{ Total } & \multirow[t]{2}{*}{$x^{2}$} & \multirow[t]{2}{*}{$d f$} & \multirow[t]{2}{*}{$P$-value } \\
\hline & Mild & $\begin{array}{l}\text { Moderate } \\
\text { or severe }\end{array}$ & & & & \\
\hline Yes & 18 (34.6\%) & 56 (77.8\%) & 74 (59.7\%) & 23.376 & 1 & $<0.001$ \\
\hline No & 34 (65.4\%) & $16(22.2 \%)$ & 50 (40.3\%) & & & \\
\hline Total & $52(100.0 \%)$ & $72(100.0 \%)$ & 124 (100.0\%) & & & \\
\hline
\end{tabular}

Abbreviations: $d f$, degrees of freedom; $\chi^{2}$, Chi-square test.

Patient opinions regarding pain management and knowledge of morphine

Sixty (48.4\%) patients believed that all pain should be treated regardless of its severity, while the other 64 (51.6\%) patients thought that not all kinds of pain deserved treatment. Moreover, 48 (38.7\%) of these 64 patients thought it was unnecessary to manage endurable pain, 19 (15.3\%) feared drug addiction, and seven (5.65\%) failed to receive any treatment due to financial issues. When asked about their knowledge of morphine, 90 (72.6\%) patients knew nothing, while the other 34 (27.4\%) recognized it. Regarding willingness to use morphine, 64 (51.6\%) patients depended on physician opinion for morphine usage, while 20 (19.4\%) and 19 (18.5\%) expressed reluctance and objection to using the drug, respectively.

\section{Pain management for patients} who reported discomfort

Among the participants, $70.2 \%$ reported moderate or severe pain to their doctors, while $11.7 \%$ did not express their pain. Regarding pain management, $52.4 \%$ of participants believed they did receive adequate attention and treatment after expressing their pain, while $14.9 \%$ claimed that no efficient pain management was conducted in time, even after reporting discomfort to doctors (Table 5). No significant correlation

Table 5 Pain intensity and management when patients reported initiatively $(n=124)$

\begin{tabular}{lll}
\hline & $\mathbf{n}$ & $\%$ \\
\hline Pain intensity when reported & & \\
No report & 15 & 11.7 \\
I-3 points & 22 & 17.7 \\
$4-6$ points & $5 \mathrm{I}$ & 41.2 \\
7-10 points & 36 & 29.0 \\
Time interval between pain being reported and treatment being received \\
No report & 15 & 11.7 \\
Immediately & 65 & 52.4 \\
I hour & 25 & 20.2 \\
I day & 4 & 3.2 \\
No treatment & 15 & 11.7 \\
\hline
\end{tabular}

was found between pain scores and the point in time when treatment was received $(P>0.05)$.

\section{Preferred postoperative pain treatment} methods among patients

When asked about their preferred methods of postoperative pain treatment, 17 out of 29 patients who had administered PCA in hospitals preferred PCA. However, most participants ( $\mathrm{n}=65,52.4 \%$ ) chose "unable to answer". Comparatively, Western medicine therapy remained the major preferred management $(n=32,25.8 \%)$.

The degree of postoperative pain relief that patients expected and satisfaction with pain control

After receiving postoperative pain treatment, most patients expected a decrease in adverse consequences caused by pain, improvement in sleep quality, and ameliorated somatic activity. About half $(n=58,46.8 \%)$ hoped that their pain level would be minimized to 0 , meaning no pain. Meanwhile, the minority $(n=7,5.6 \%)$ thought it would be adequate for treatment to reduce severe pain to a moderate intensity. In general, study participants were satisfied with their pain treatment since they achieved their expected effects. However, 20.2\% of patients ( $\mathrm{n}=25)$ were unsatisfied with their pain treatments and continued to suffer from physical and mental ailments caused by postoperative pain even after treatment.

\section{Discussion}

\section{Influence of postoperative pain on patients' QOL}

Postoperative pain management is an essential component of care for which operating surgeons, anesthetists, and nurses strive. Wu et al discovered that an increase in postoperative pain decreased the patient's QOL within 2 weeks of surgery. ${ }^{8}$ Similarly, our research indicated that postoperative pain produced negative effects on the patients' QOL, due to debilitation, nausea, vomiting, dizziness, and headache. Nausea and vomiting are particularly troublesome, as they may lead to 
tension on the operative site, causing tears and infection at the incision suture site. Furthermore, moderate and severe pain can even lead to sleep and mental disorders. Sleep and mental disorders may aggravate the postoperative pain and prolong healing time. In addition, stress from pain can elevate catabolism, increase cardiac load, weaken immune system function, cause abnormal coagulation function, and lead to functional disturbance of numerous body systems. ${ }^{9,10}$ Furthermore, inadequate pain management can decrease the patient's QOL, prevent recovery from diseases, and prolong the hospital stay. ${ }^{11}$ Therefore, postoperative pain management should be prioritized by hospital personnel and patients.

\section{Methods and effects of postoperative pain management}

PCA, temporary analgesia, or a combination of the two was commonly used in Hunan provincial-level hospitals to treat postoperative pain. The survey revealed that pain treatment was irregular and was not performed until patients proactively requested pain management when suffering from severe pain. In total, $91.4 \%$ of patients suffered from moderate or severe pain after an operation, which was significantly higher than what previous research has shown. ${ }^{3,4}$ Although $82.8 \%$ of participants claimed that their postoperative pain had been relieved or disappeared 3 days after their operation, approximately $20 \%$ of participants were dissatisfied with their pain management. The aforementioned results demonstrate the inadequate use of strong opioids and poorly timed pain management methods, thus emphasizing the need to improve and standardize postoperative pain management plans in Chinese hospitals.

Strassels et al believe that correct analgesic usage does not develop into drug addiction and argued that on-time delivery, rather than on-demand delivery can lower the risk of addiction. ${ }^{2}$ Moreover, previous research proves that the postoperative pain is associated not only with peripheral sensitization but also with central sensitization. This means that postoperative pain management aims to inhibit or reverse the change of central neuronal excitability, instead of merely removing physiological and pathological pain. ${ }^{12}$ The current postoperative pain management plans provided in Hunan provincial-level hospitals are still far from meeting patient expectations, and there is a need to strengthen the pain education for medical staff.

\section{Patient knowledge and opinions regarding postoperative pain and its management}

Patient knowledge and opinions regarding postoperative pain and its management directly influence the implementation and efficacy of treatment; this revelation may change the way physicians approach postoperative pain and affect the strategies used for postoperative pain treatment. The survey results revealed reluctance to pain management drugs, as $51.6 \%$ of patients thought that not all kinds of pain deserved treatment, and $38.7 \%$ said it was unnecessary to manage endurable pain, while $15.3 \%$ were afraid of drug addiction.

Lack of enthusiasm for pain management may be a consequence of traditional Chinese culture and national history. Chinese people have long inherited the spirit of ancient precepts to bear hardships, which discourages them from searching for treatment and proactively reporting their pain during hospitalization. Meanwhile, the Chinese public has a poor understanding of postoperative pain, revealing the importance of promoting education on pain. According to the survey, $72.6 \%$ of patients knew nothing about morphine, while the remaining $27.4 \%$ understood morphine to be an affordable medicine with a strong analgesic function, great risk of addiction, and severe side effects. Still, $18.5 \%$ of patients strongly rejected morphine usage due to the drug's reputation in the People's Republic of China as a result of the Opium Wars in the 1800s. While the Chinese public is familiar with opioids, the demonstrated poor recognition of morphine in this survey may result from the infrequent use of the word "morphine". Furthermore, the widespread fear of drug addiction and morphine's adverse effects is likely to be influenced by the People's Republic of China's national history.

In addition, Lin found that family bonds in the People's Republic of China played a key role in the process of pain management, as family opinions may affect patient concerns regarding drug addiction. ${ }^{13}$ In reality, the risk of opioid addiction during postoperative pain management is quite low. Porter and Jick reported that among 11,882 patients who received at least one kind of strong opioid treatment, only four developed psychic dependence. ${ }^{14}$ Opioids are the first choice for treating severe pain, and the analgesic effect of morphine has been widely recognized, which indicate the extent of Chinese patients' misunderstanding of opioids. Therefore, it is urgent for medical staff to strengthen patient education on analgesics and inform patients that analgesics rarely cause addiction, will not prevent wound healing, and promote quick rehabilitation. Moreover, postoperative pain management plans can encourage patients to actively cooperate with doctors in their treatment to relieve postoperative pain.

As shown in the survey, $46.8 \%$ of participants hoped that treatment could remove their pain and expected treatment to reduce pain's adverse effects by improving sleep quality and increasing somatic activities. Of the study participants, 
the minority of patients failed to report their pain, while the majority reported moderate or severe pain. The failure to report pain in a timely manner may have resulted from the poor knowledge of analgesic treatment and limited economic resources presented by patients. Doctors should encourage patients who have undergone operations to report their postoperative pain in order to get treatment in sufficient time. On the other hand, $14.9 \%$ of patients did not receive any treatment in a timely way, even though they had reported their pain, and pain evaluations were not performed routinely on these postoperative patients. Thus, it is clear that medical staff should place more emphasis on postoperative pain when planning and administering treatment.

The study has limitations in that the sample was taken from only three provincial-level hospitals; however, these hospitals are affiliated with Xiangya Medical School of Central South University, which is academically renowned for the research it produces. Moreover, the surveyed hospitals are the largest in Hunan Province, as each one houses over 2,000 beds. Thus, these three hospitals are representative of provincial-level hospitals in the People's Republic of China to some extent.

\section{Conclusion}

This survey objectively reflects postoperative pain management, current status of pain control, and patient knowledge and opinions regarding postoperative pain and its treatment in Hunan's provincial-level hospitals, which are representative of the People's Republic of China's provincial-level hospitals. Although postoperative pain management can relieve pain for many patients, the People's Republic of China trails far behind in this respect to its developed peers because of inadequate attention paid to postoperative pain, lack of routine pain assessments after operations, and unstandardized pain management methods. Inadequate knowledge of postoperative pain and poor communication with medical personnel, as demonstrated by patients, result in the delay of pain management. In addition, patient misunderstanding of analgesics leads to ineffective pain treatment. In order to improve the level of postoperative pain management in the People's Republic of China, relieve patients' pain, and improve patient QOL, a pain education campaign should be prioritized and implemented within hospitals to educate medical staff, patients, and their families.

\section{Disclosure}

The authors declare no conflicts of interest in this work.

\section{References}

1. International Association for the Study of Pain - IASP Taxonomy [webpage on the Internet]. Washington: International Association for the Study of Pain. Available from: http://www.iasp-pain.org/AM/Template. cfm?Section=Pain_Definitions. Accessed May 22, 2012.

2. Strassels SA, McNicol E, Suleman R. Postoperative pain management: a practical review, part 1. Am J Health Syst Pharm. 2005;62(18): 1904-1916.

3. Maier C, Nestler N, Richter H, et al. The quality of pain management in German hospitals. Dtsch Arztebl Int. 2010;107(36):607-614.

4. Apfelbaum JL, Chen C, Mehta SS, Gan TJ. Postoperative pain experience: results from a national survey suggest postoperative pain continues to be undermanaged. Anesth Analg. 2003;97(2):534-540.

5. Melzack R. The McGill Pain Questionnaire: major properties and scoring methods. Pain. 1975;1(3):277-299.

6. Garwood J. A guide to research on the Rorschach prognostic rating scale. J Pers Assess. 1977;41(2):117-119.

7. Pain Management Guideline. Hamilton: Health Care Association of New Jersey. Available from: http://www.hcanj.org/files/2013/09/hcanjbp_painmgmt2_3.pdf. Accessed July 18, 2006.

8. Wu CL, Naqibuddin M, Rowlingson AJ, Lietman SA, Jermyn RM, Fleisher LA. The effect of pain on health-related quality of life in the immediate postoperative period. Anesth Analg. 2003;97(4): 1078-1085.

9. Page GG. The immune-suppressive effects of pain. Adv Exp Med Biol. 2003;521:117-125.

10. Joshi GP, Ogunnaike BO. Consequences of inadequate postoperative pain relief and chronic persistent postoperative pain. Anesthesiol Clin North America. 2005;23(1):21-36.

11. Pyati S, Gan TJ. Perioperative pain management. CNS Drugs. 2007;21(3):185-211.

12. Qi QL, Yang JL. Postoperative analgesia. In: Yang JL, Li ZQ, Han JX, editors. Acute Pain Therapeutics. Beijing: People's Military Medical Press; 2006:495.

13. Lin CC. Barriers to the analgesic management of cancer pain: a comparison of attitudes of Taiwanese patients and their family caregivers. Pain. 2000;88(1):7-14.

14. Porter J, Jick H. Addiction rare in patients treated with Narcotics. NEngl J Med. 1980;302(2):123.
Patient Preference and Adherence

\section{Publish your work in this journal}

Patient Preference and Adherence is an international, peer-reviewed, open access journal focusing on the growing importance of patient preference and adherence throughout the therapeutic continuum. Patient satisfaction, acceptability, quality of life, compliance, persistence and their role in developing new therapeutic modalities and compounds to

\section{Dovepress}

optimize clinical outcomes for existing disease states are major areas of interest. This journal has been accepted for indexing on PubMed Central. The manuscript management system is completely online and includes a very quick and fair peer-review system. Visit http://www.dovepress.com/ testimonials.php to read real quotes from published authors. 